\title{
BRDF of human skin in the visible spectrum
}

\begin{tabular}{|c|c|}
\hline Journal: & Sensor Review \\
\hline Manuscript ID & SR-11-2016-0258.R1 \\
\hline Manuscript Type: & Original Manuscript \\
\hline Keywords: & Reflectance, Spectral analysis, Machine vision, Rendering, BRDF \\
\hline
\end{tabular}




\begin{abstract}
Purpose - Significant research has been carried out in terms of development of new BRDF instruments, however there is still little research available regarding spectral BRDF measurements of human skin. This study aims to investigate the variation in human skin reflectance using a new fibre optic based spectral-BRDF measurement device.

Design/methodology/approach - Design of our system mainly involves use of multiple fibre optics to illuminate and detect light reflected from a sample, while a hemispherical dome was 3D printed to mount the fibres at various slant/tilt angles. In order to investigate the spectral differences in BRDF of human skin, 3 narrowband filters in the visible spectrum were used while measurements were taken from the back of the hand for Caucasian and Asian skin types.

Findings - The experiments demonstrate that the BRDF of human skin varies with wavelengths in the visible spectrum and it is also different for Caucasian and Asian skin types. Both skin types exhibit off-specular reflection with increase in angle of incidence and show less variation with respect to viewing angles when the angle of incidence is normal to the surface. Research implications - A database of spectral BRDF measurements of human skin will not only help in creating realistic skin renderings but also in development of novel skin reflectance models for biomedical and machine vision applications. The measurements would also provide means to validate the predictions from existing light transport/ spectral simulation models for human skin and will ultimately help in the accurate diagnosis and simulation of various skin disorders.

Originality/value - Our proposed system provides fast scatter measurements by utilising multiple fibres to detect light simultaneously at different angles while also allowing easy switching between incident light directions. Due to its flexible design and contact based measurements, the device is independent of errors due to sample movements and does not require any image registration. Also, measurements taken from the device show that the BRDF of skin varies significantly in the visible spectrum and it is different for Caucasian and Asian skin types.
\end{abstract}

Keywords Machine vision, Reflectance, Spectral analysis, Rendering, BRDF

Paper type Research paper

\title{
1. Introduction
}

As light interacts with a surface, some of it is transmitted through the material, some gets absorbed and the rest is reflected. This complex light interaction with real world materials depends on the physical properties of light and the composition and physical properties of materials. Because of the variation in material properties and composition, surfaces can appear to be shiny, diffuse, opaque, translucent or a combination of these. Also, there can be variation due to wavelength as some materials reflect/ absorb light differently at different wavelengths. The Bidirectional Reflectance Distribution Function (BRDF) is used to describe this complex light interaction with surfaces and it shows the distribution of reflected light as the direction of incident illumination varies relative to the surface orientation.

The BRDF describes how a surface reflects light when illuminated from different directions. In its simplest form, it is described using 5 variables: incidence and reflected light direction defined in terms of slant and azimuth angles and a term for wavelength. The BRDF has a dimensionless unit of steradian inverse and It is a ratio of differential radiance to the incident irradiance on a surface patch (equation (1)):

$$
\operatorname{BRDF}\left(\theta_{i}, \varphi_{i}, \theta_{o}, \varphi_{o}, \lambda\right)=\frac{d L_{o}\left(\theta_{o}, \varphi_{o}, \lambda\right)}{d E_{i}\left(\theta_{i}, \varphi_{i}, \lambda\right)}=\frac{d L_{o}\left(\theta_{o}, \varphi_{o}, \lambda\right)}{L_{i}\left(\theta_{i}, \varphi_{i}, \lambda\right) \cos \theta_{i} d \omega_{i}}
$$

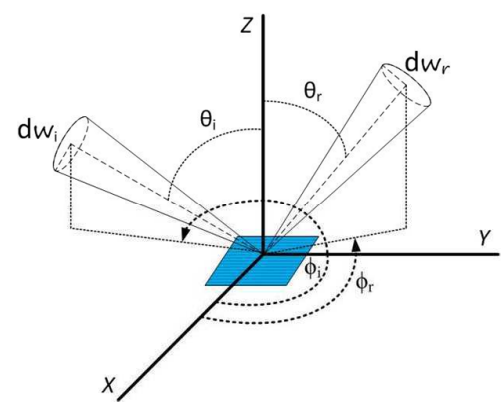

Figure 1 BRDF description in terms of incident and reflected rays. 
The Incidence and viewing angles are represented in spherical coordinates and are measured with respect to surface normal. A visual representation of BRDF measurements and the description of variables involved is presented in Figure 1 and Table 1.

Table 1 Description of BRDF parameters.

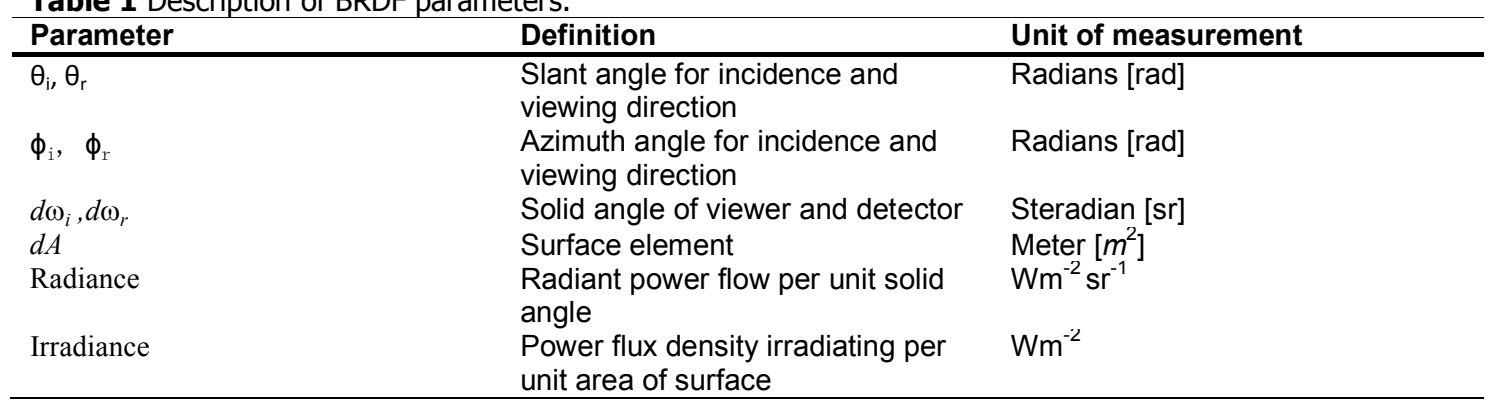

The capture of dense BRDF measurements is a complex and time consuming process especially when the surface exhibits varying reflective properties with varying wavelength. For each incident light direction, it requires measurement of reflected light in all possible viewing directions leading to large storage requirements. However, BRDF measurements find their usefulness in range of applications such as realistic image synthesis, simulation models (Baranoski et al. 2014), reflectance and light transport models (Li \& Carmen 2009), remote imaging, photo realistic rendering, cosmetics, paint and coatings analysis (Seubert 2016; Gatebe \& King 2016; Boher et al. 2015) and are often used as a validation tool (Westin et al. 2004).

Human skin is made up of a multi-layered structure with complex optical properties. It's reflectance is known to vary with wavelength and skin type. However, little work has been done regarding spectral BRDF measurements of human skin. The measurement of spectral BRDF of skin is not only useful for shape for shading algorithms but also for biomedical applications. These measurements can be used to produce novel skin reflectance models and can also be used for evaluation of existing models. Several light transport models exist (Baranoski et al. 2014) that predict skin reflectance using biologically inspired parameters. A database of these spectral measurements could be used for quantitative evaluation of predictions made by these models.

The inspiration behind most of the BRDF measurements for human skin is based on producing realistic renderings and hence are based on RGB triplets only. Also, it is believed that the BRDF of skin does not vary considerably in the visible part of the spectrum (especially in RGB region). In this paper, we propose the use of a fibre optic based device that can be used to create a large database of spectral-BRDF measurements of human skin and we also present measurements of different skin types over narrowband wavelengths to show variations in BRDF in the visible spectrum.

\section{Review of existing techniques}

BRDF is traditionally measured using a gonioreflectometer device ( $\mathrm{Li}$ et al. 2006). The device mainly consists of three basic components: a sample holder, detector and an illumination source. The illumination and detector are designed to move over the entire hemisphere around the sample to capture reflected light for a specific incident light direction. Gonioreflectometers can not only capture dense spectral measurements but can also provide higher spatial coverage over the complete BRDF space. The detector used in these devices is usually based on spectroradiometers/diode-arrays and can provide better precision/sensitivity to light variation as compared to the image based techniques. Since these devices make use of multi-axis motorised stages/robots (Belcour et al. 2014; Hünerhoff et al. 2006) to move around the light source, detector and sample, it makes the capture process slow (tens of hours) and impractical for human skin measurements.

To speed up measurements, several alternative designs have been proposed which mainly involve reducing the motorised components and speeding up acquisition time by detecting light simultaneously at all angular locations. Dana came up with a concave mirror design for simultaneous measurement of spatially varying BRDF(Dana 2001). A half silvered hemisphere along with a fish eye lens was used by Ward to capture the outgoing reflected light instantaneously in a single image, however the design still required motorised parts for changing the light source direction (Ward 1992). (Marschner et al. 1999; Kim et al. 2009) used image based BRDF measurement technique and reduced the capture time by using curved samples with different orientations. Given the 3D model of the sample, the illumination and viewing directions relative to a number of different surface normals allowed them to capture the BRDF in relatively less time than traditional gonioreflectometer measurements.

Non-spectral BRDF measurements are done in abundance and are also available for human skin, while limited research is available regarding spectral BRDF measurements. An LED based device was proposed by Ben (BenEzra et al. 2008) that utilises the ability of LED's to act as both detector and illuminator. The LEDS also has an added advantage that they provide a higher dynamic range compared to the CCD based designs and are also low cost. (Sun \& Wang 2005) proposed two systems (sample fixed, sample rotation) based on a typical 
gonioreflectometer design for handling different sample sizes. A modified image based measurement was proposed by (Hullin et al. 2010), their design involved a rotary stage, camera and light source with an added tunable filter in front of camera. The device was used to measure BRDF of a variety of fluorescent materials like automobile paints, fabrics etc. .For taking spectral BRDF measurements of human skin, (Koch 2011) used the CASI ${ }^{\circledR}$ scatterometer device to take measurements at 2 spectral bands while an image based multicamera system was developed by (Jeffrey R. Bintz et al. 2016) for taking measurements at 4 spectral bands in the VIS/NIR region. However, for both systems, limited sample measurements were made available for different skin types.

Most BRDF measurement devices have one thing in common, they have to move the detector to cover a range of viewing directions making the process slow and applicable to static objects only. The device presented in this paper uses fixed detectors at various scatter angles thereby reducing the capture time by taking measurements instantaneously. The motivation behind most of the BRDF measurement of human skin involves computer graphics and rendering applications. Although there are a few cases where spectral BRDF measurements (over a few wavelengths) were taken for human skin, but there is still lack of spectral BRDF measurements for different ethnicities, age groups and gender. We present a prototype device that has the potential to provide reliable spectral BRDF information for human skin and can be used to create a large database of human skin measurements.

\section{Prototype Implementation}

The device is based on the concept of using fibre optics as both illuminators as detectors. An assembly in the form of a hemispherical dome was created using 3D printing to mount the fibres. The CAD model of the dome in Figure 2(b) shows holes created at precise locations to illuminate the sample and to record the light reflected from it. Since we are only interested in the forward scatter measurements of skin, fibres were added at 0, 2.5, $7.5,12.5,17.5,25,30,37.5,45,52.5,57.5,67.5,72.5$, and 75 degrees angles to measure the scattered light from the sample. Multi-mode plastic optical fibres were used with both ends of the fibre machined polished to minimise any light losses. The size of the $3 \mathrm{D}$ printed dome was set at $25.4 \mathrm{~cm}$ diameter, while a circular opening of $3.5 \mathrm{~cm}$ diameter was created at the centre of the dome for illuminating the samples. In order to collimate the light over the samples, ball lens were also attached at the end of the fibres entering the dome.

Instead of making the illumination direction fixed, we can change the direction of illumination by dividing the light feed into the dome in two stages. Stage-1 consists of fibre's mounted over a high-power LED and the other end is attached to a ball lens coupler. The ball lens takes the light from stage- 1 fibre and transmits it to the stage-2 fibre which is ultimately taken inside the dome. Diving the light feed into two stages allows us to change the direction of illumination easily by moving the stage- 1 fibre over a series of fibres located in the docking area in stage-2. A motorised linear stage was used to move the stage-1 fibres and a graphical representation of this twostage light feed is shown in Figure 2 (a) and (c). The advantage of using fixed individual fibres at different viewing angles allows us to capture reflectance instantaneously and makes it robust to sample movement and faster than traditional gonioreflectometer measurements.

Figure 2 (a) Proposed fibre optic based BRDF measurement device (b) CAD model of 3D printed dome (c) Magnified (CAD model) of stage- 1 and 2 of light feed.

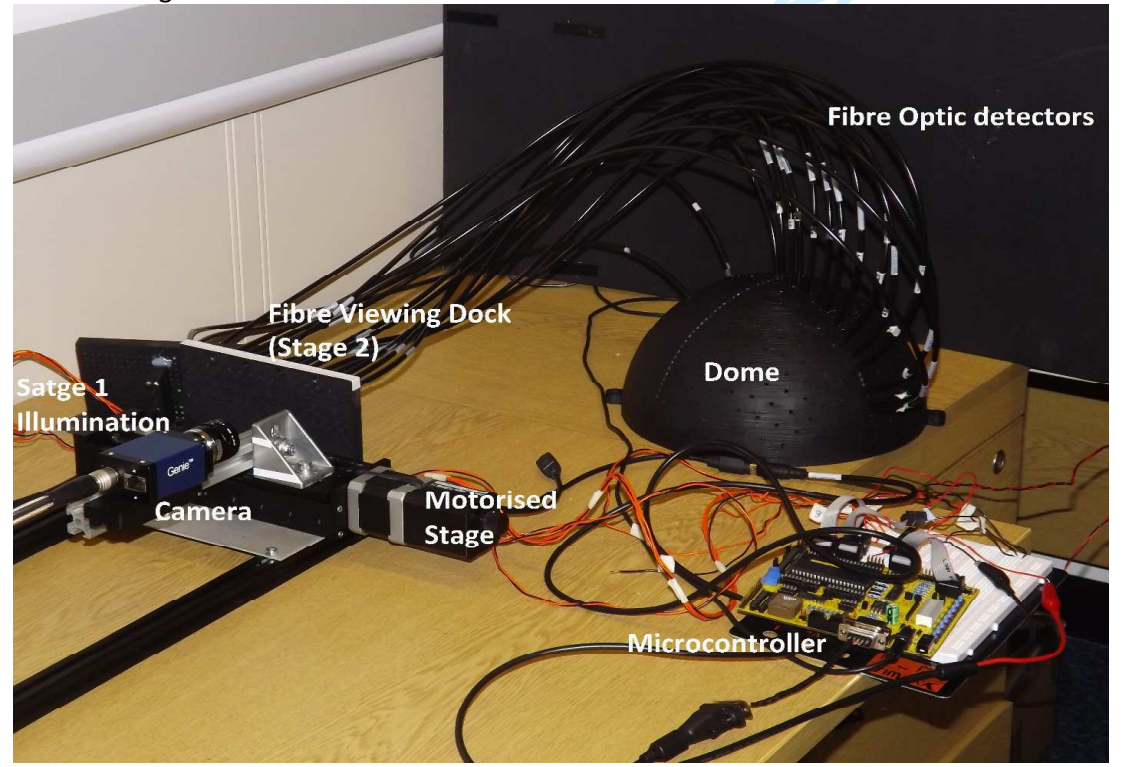

(a) 


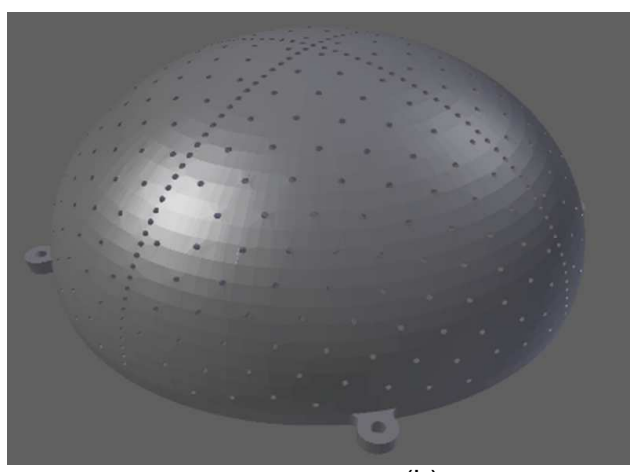

(b)

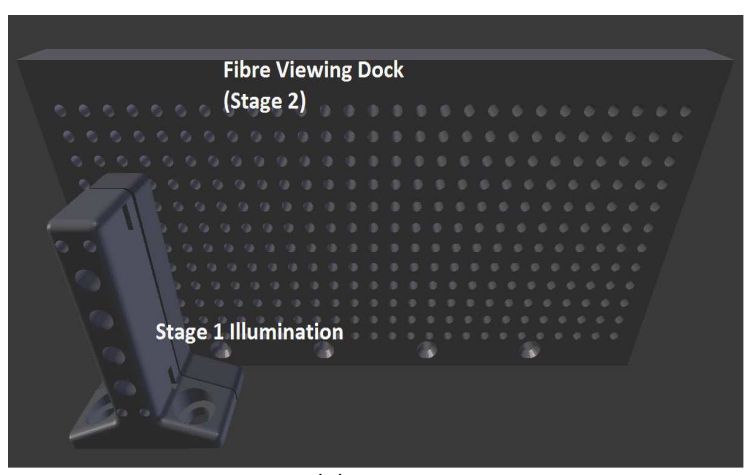

(c)

For spectral BRDF measurements, narrowband filters with central wavelengths at $450 \mathrm{~nm}, 530 \mathrm{~nm}$ and $630 \mathrm{~nm}$ were added with FWHM bandwidth of $27 \mathrm{~nm}, 25 \mathrm{~nm}$ and $34 \mathrm{~nm}$ respectively and transmission $\geq 80 \%$. High power LEDs were used as source of illumination while a 12-bit monochrome camera was used to collect the light from all the stage-2 fibres attached to the viewing dock. Absolute radiometric calibration was performed for each narrow band filter to convert sensor digital numbers (DN) to spectral radiance.

Measurements can be taken by placing the sample in the opening provided at the centre of the dome. Light is then fed in to the dome through stage- 1 and 2 fibres and sample is illuminated from a particular light direction. All scatter light is picked up by fibres at different viewing angles and is ultimately captured by the camera. Incident light direction is then changed by moving stage-1 fibre over stage- 2 viewing dock using the motorised linear stage. The measurements are then repeated until all incident light direction have been covered. The camera is connected to the PC where all the data is transferred and processed while the microcontroller is responsible for hardware triggering and synchronisation of camera, LEDs and the control of motorised stage.

\section{Experiments and discussion}

For practical measurements, the BRDF is defined using the components such as incident and reflected power $\left(P_{i}\right.$ and $P_{s}$ respectively), solid angle $(\Omega)$ due to the size of detector, distance to detector from sample $(R)$ and the scatter angle $\left(\theta_{s}\right)$. For a given incidence and scatter angle, the BRDF was calculated using:

$$
B R D F=\frac{\frac{P_{s}}{\Omega}}{P_{i} \cos \theta_{s}}
$$

For each measurement, a dark image was taken along with an image without the sample in place to remove any stray light in receiving fibres. Before taking any measurements for skin, we validated the measurements from our device by taking BRDF measurements of a spectralon white sample. Measurements were taken at two incidence angles ( 0 and 60 DEG) and are shown in Figure 3.

Figure 3 (a), (b)The BRDF of Spectralon white at 0 and 60 incidence angles (dotted vertical line shows angle of incidence).

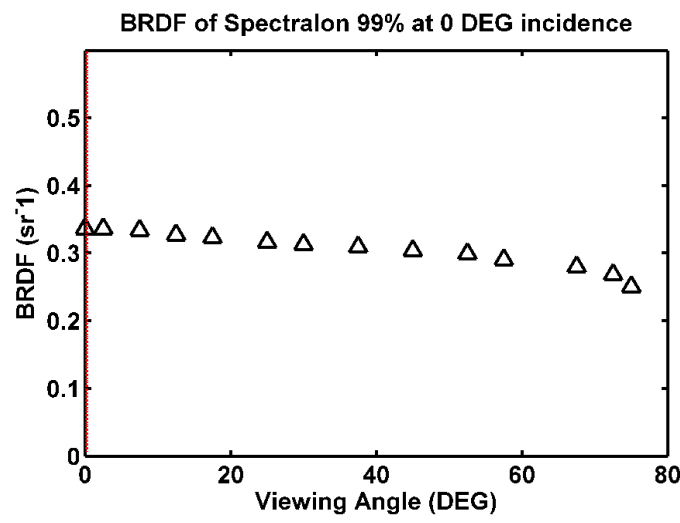

(a)

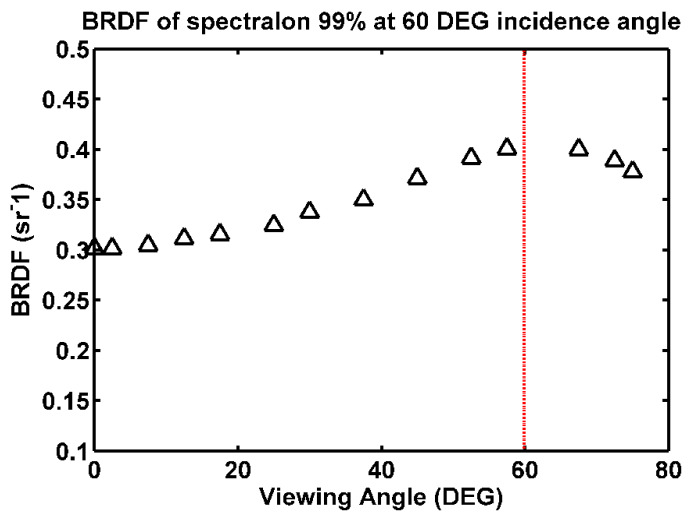

(b) 
Using our device, we took measurements for Caucasian and Asian skin types at three wavelengths in the visible spectrum. A total of six subjects were selected for this experiment, among them 3 were Caucasians and the other 3 Asians. Since we are only interested in the difference in BRDF for these two skin types, we took measurements for forward scatter only and for two incidence angles at 0 and 60 degrees. Measurements were taken by placing the back of hand at the sample area of the device. Figures 4 and 5 show BRDF measurements taken and are plotted as a function of viewing angle and wavelength.

The measurements show considerable difference in BRDF for both skin types as the BRDF changes not only with wavelength but also with angle of incidence. For each wavelength, BRDF seems to be more close to Lambertian at normal incidence angles. However, as the angle of incidence increases, there is an increase in reflectance at higher viewing angles. This variation is true for both skin types as shown in Figures 4 and 5 . Due to higher melanin content in Asian skin type, there is a decrease in reflectance compared to Caucasian skin type which can be seen at all wavelengths and for both angle of incidence. However, both skin types have one thing in common, both show off-specular reflection at $60 \mathrm{deg}$ incidence angle and the effect is same irrespective of wavelength. This off-specular reflection behaviour has also been observed for human skin when the reflectance's were taken as an aggregate over the visible spectrum (Marschner et al. 1999).

Figure 4 (a), (b)BRDF of Asian skin type at 0 and 60 degree's incidence angles (dotted vertical line shows angle of incidence).

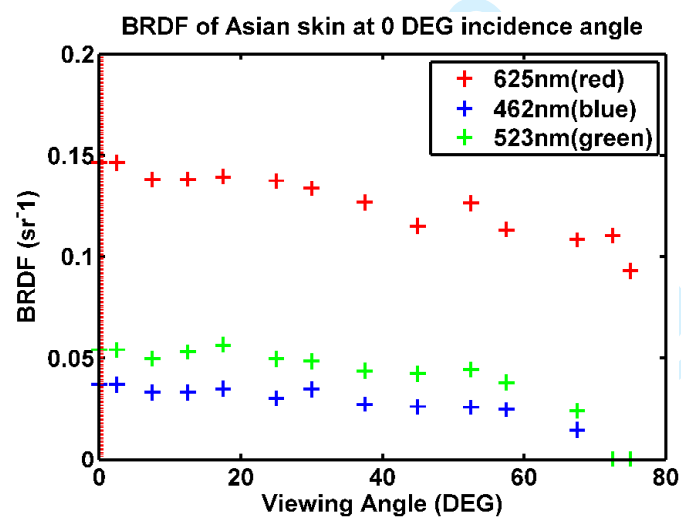

(a)

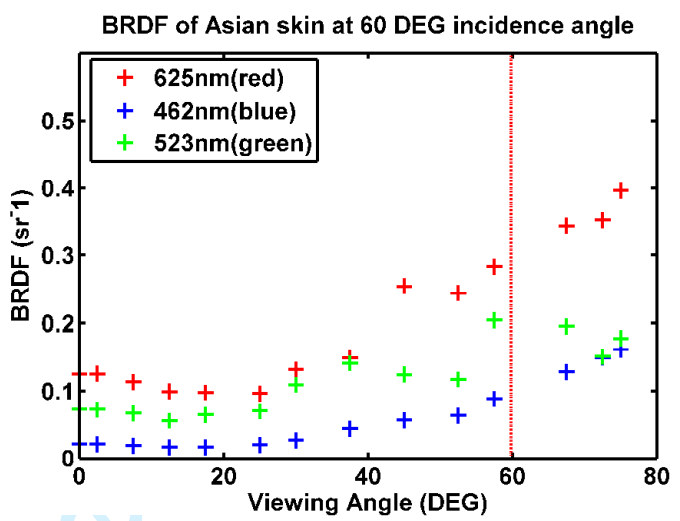

(b)

Figure 5 (a), (b)BRDF of Caucasian skin type at 0 and 60 degree incidence angles (dotted vertical line shows angle of incidence).

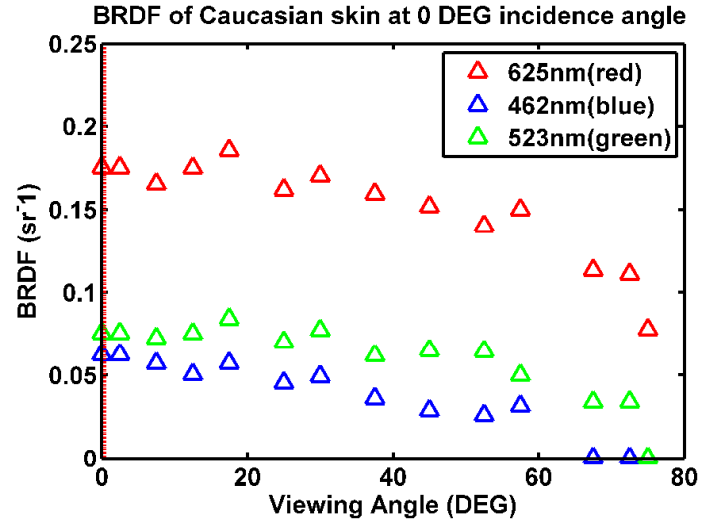

(a)

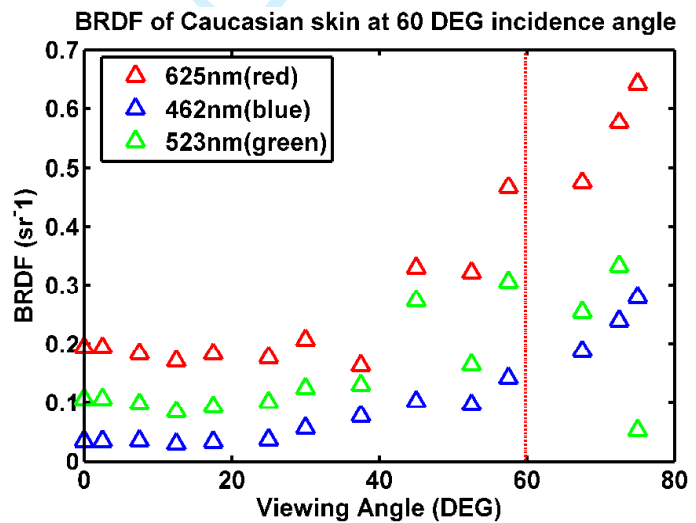

(b)

The combination of lightweight 3D printed dome and fibre optics makes for a flexible design and allows it to be placed in-contact over the sample. Since the dome is always in contact with the sample, it moves as the sample underneath starts moving. This feature is particularly useful for human skin where it is difficult to stay still for long periods and makes the whole measurement process very simple. When the dome comes in to contact with human skin, it moves in synchronous to skin movements whether it is a large or an involuntary small movement. Another advantage of this dome based design is that the illumination directions are not fixed and can be easily 
changed using the stepper motor, while the receiving fibres can capture light at all viewing directions simultaneously hence reducing the measurement time considerably.

\section{Conclusion}

A fibre optic based spectral-BRDF measurement device is presented in this paper. Although the device was used for measurement of skin BRDF at narrowband wavelengths in the visible spectrum, it can be used for measurement of other materials. It provides simultaneous measurements of scattered light at varying viewing angles while its motorised light feed system allows easy switching of incidence angle of light. Since the dome base detector is always in contact with the skin sample, it is not effected by movements between scans and also does not require any registration in postprocessing.

Most BRDF measurements of human skin are taken as an aggregate over the visible spectrum and very limited research is available regarding spectral BRDF of human skin. The spectral measurements taken from our device show clear differences in BRDF for both Caucasian and Asian skin types while also showing some similar characteristics in terms of off-specular reflection behaviour with increase in angle of incidence. The proposed device can be easily modified to measure BRDF for a range of wavelengths by simply changing the filter. In future, we are looking to expand the measurements for a wider range of incoming and viewing angles, for a larger set of wavelengths including the infrared region and for a variety of age groups/gender/ethnicities.

\section{References}

Baranoski, G.V.G., Chen, T.F. \& Krishnaswamy, A., 2014. Mul tilayer Modeling of Skin Color and Translucency. In Computational Biophysics of the Skin. pp. 978-981.

Belcour, L. et al., 2014. BRDF Measurements and Analysis of Retroreflective Materials. Journal of the Optical Society of America A, 31(12), pp.2561-2572.

Ben-Ezra, M. et al., 2008. An LED-only BRDF measurement device. In Computer Vision and Pattern Recognition. IEEE, pp. 18.

Boher, P. et al., 2015. New generation of Fourier optics instruments for fast multispectral BRDF characterization. Measuring, Modeling, and Reproducing Material Appearance 2015, 9398(October 2016), pp.1-12.

Dana, K.J., 2001. BRDF/BTF measurement device. In ICCV. IEEE, pp. 460-466.

Gatebe, C.K. \& King, M.D., 2016. Airborne spectral BRDF of various surface types (ocean, vegetation, snow, desert, wetlands, cloud decks, smoke layers) for remote sensing applications. Remote Sensing of Environment, 179(October), pp.131-148.

Hullin, M.B. et al., 2010. Acquisition and Analysis of Bispectral Bidirectional Reflectance and Reradiation Distribution Functions. Acm Tog, 29(4), p.97:1--97:7.

Hünerhoff, D., Grusemann, U. \& Höpe, a, 2006. New robot-based gonioreflectometer for measuring spectral diffuse reflection. Metrologia, 43, pp.S11-S16.

Jeffrey R. Bintz et al., 2016. A novel image-based BRDF measurement system and its application to human skin. In Reflection, Scattering, and Diffraction from Surfaces.

Kim, D.B. et al., 2009. High-dynamic-range camera-based bidirectional reflectance distribution function measurement system for isotropic materials. Optical Engineering, 48(9), p.93601.

Koch, B.M., 2011. A MULTISPECTRAL BIDIRECTIONAL REFLECTANCE DISTRIBUTION FUNCTION STUDY OF HUMAN SKIN FOR IMPROVED DISMOUNT DETECTION. Air Force Institute of Technology.

Li, H. et al., 2006. Automated three-axis gonioreflectometer for computer graphics applications. Optical Engineering, 45(4), pp.1-11.

Li, L. \& Carmen, S.L.N., 2009. Rendering human skin using a multi-layer reflection model. International Journal of Mathematics and Computers in Simulation, 3(1), pp.44-53.

Marschner, S.R. et al., 1999. Image Based BRDF Measurement Including Human Skin. In Eurographics Workshop on Rendering. pp. 131-144.

Seubert, C.M., 2016. The Appearance of Platelet-Polymer Composite Coatings: Microstructural Characterization, Hybrid Modeling, and Predictive Design. University of Michigan. 
1

2

3

4

5

6

7

8

9

10

11

12

13

14

15

16

17

18

19

20

21

22

23

24

25

26

27

28

29

30

31

32

33

34

35

36

37

38

39

40

41

42

43

44

45

46

47

48

49

50

51

52

53

54

55

56

57

58

59

60

Sun, Y. \& Wang, Q., 2005. Spectral BRDF Measurement and Data Interpolation. In 13th Color and Imaging Conference. pp. 114-119.

Ward, G., 1992. Measuring and modeling anisotropic reflection. ACM SIGGRAPH Computer Graphics, (July), pp.265-272.

Westin, S.H.H., Li, H. \& Torrance, K.E.E., 2004. A comparison of four brdf models. Proc. Eurographics Symposium on Rendering, pp.1-10. 


\begin{tabular}{|c|c|c|}
\hline Parameter & Definition & Unit of measurement \\
\hline$\overline{\theta_{i}, \theta_{\mathrm{r}}}$ & $\begin{array}{l}\text { Slant angle for incidence and } \\
\text { viewing direction }\end{array}$ & Radians [rad] \\
\hline$\phi_{i}, \phi_{r}$ & $\begin{array}{l}\text { Azimuth angle for incidence and } \\
\text { viewing direction }\end{array}$ & Radians [rad] \\
\hline$d \omega_{i}, d \omega_{r}$ & Solid angle of viewer and detector & Steradian [sr] \\
\hline$d A$ & Surface element & Meter $\left[m^{2}\right]$ \\
\hline Radiance & $\begin{array}{l}\text { Radiant power flow per unit solid } \\
\text { angle }\end{array}$ & $\mathrm{Wm}^{-2} \mathrm{sr}^{-1}$ \\
\hline Irradiance & $\begin{array}{l}\text { Power flux density irradiating per } \\
\text { unit area of surface }\end{array}$ & $W^{-2}$ \\
\hline
\end{tabular}




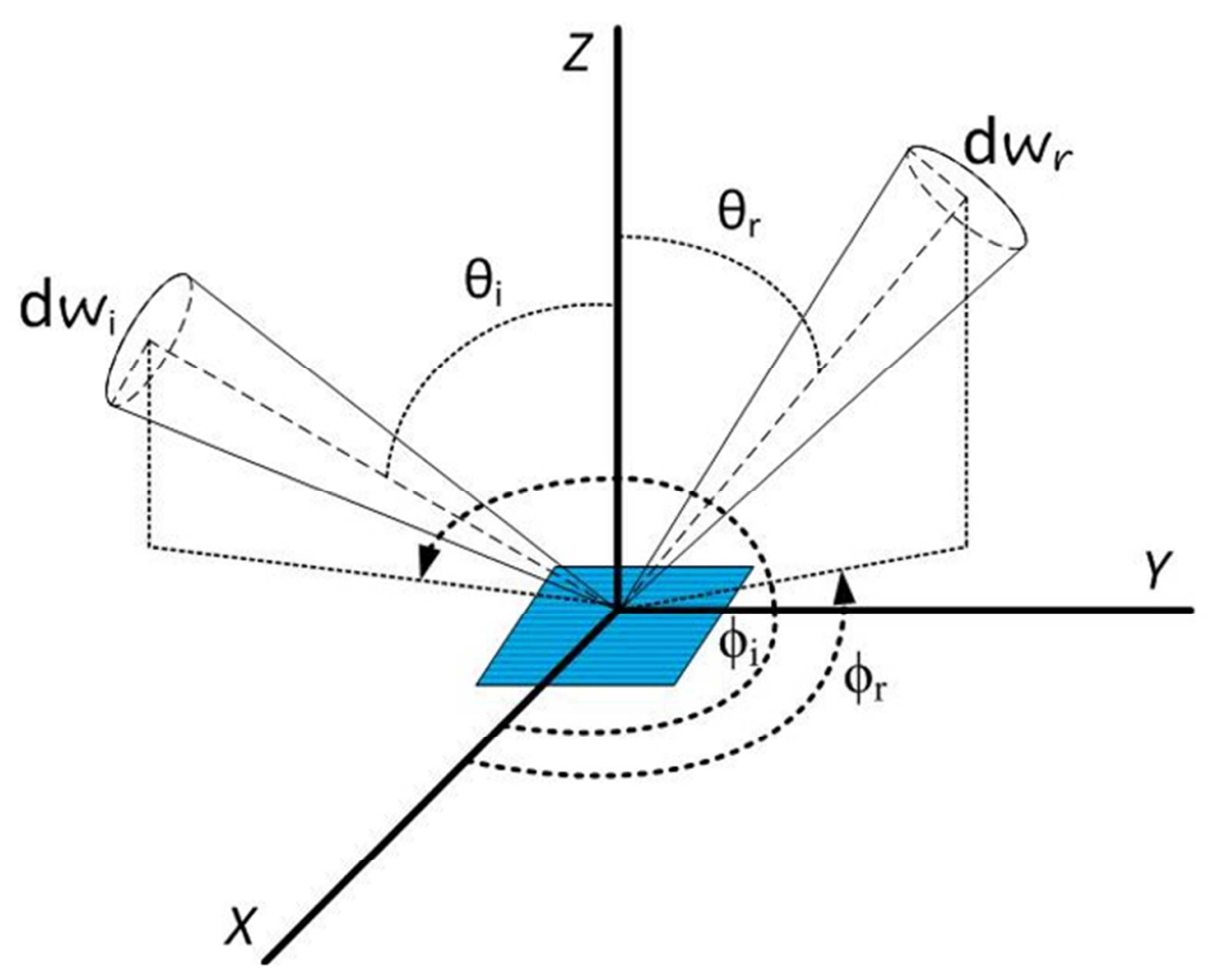




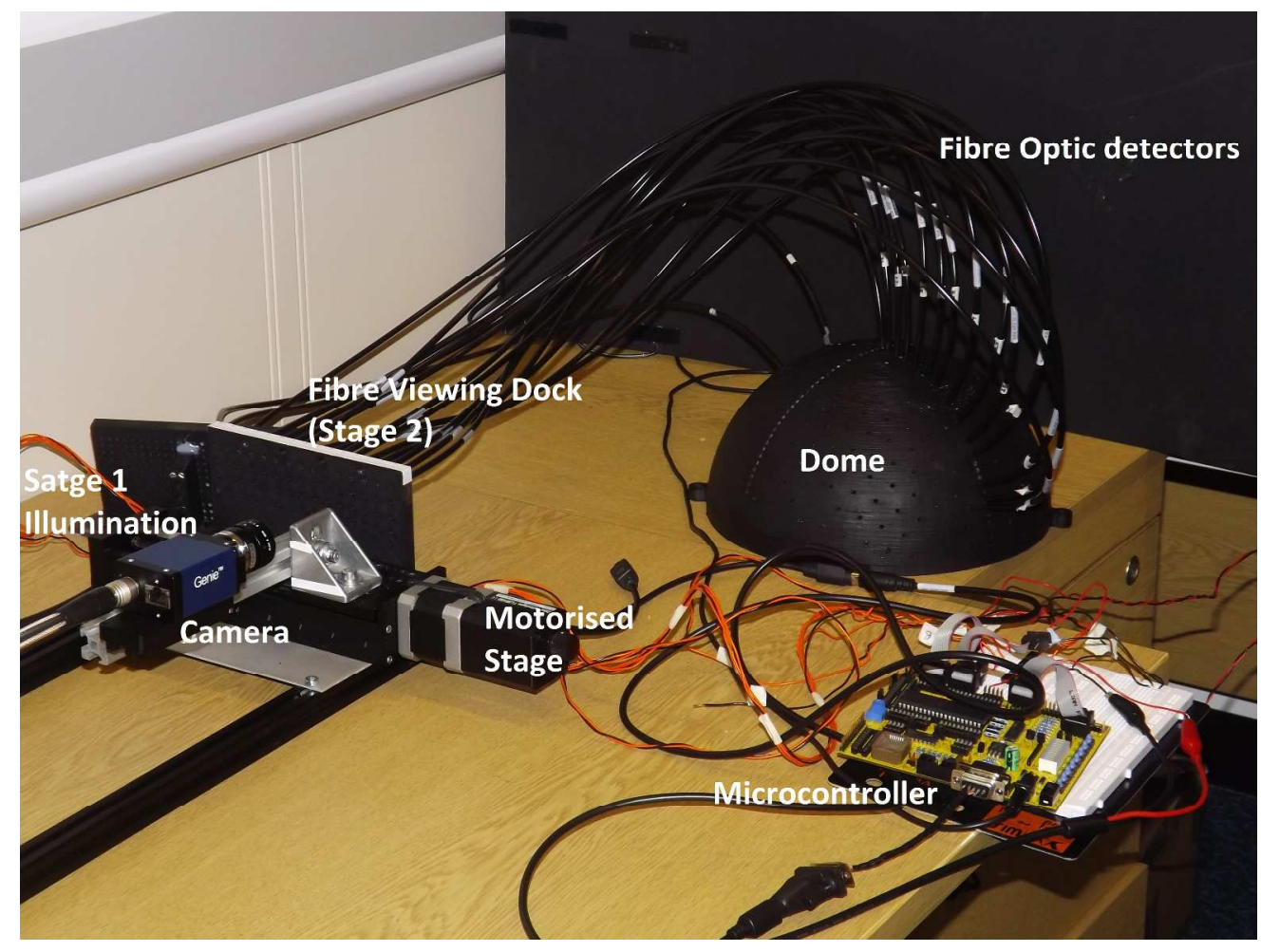

1512x1134mm (72 x 72 DPI) 


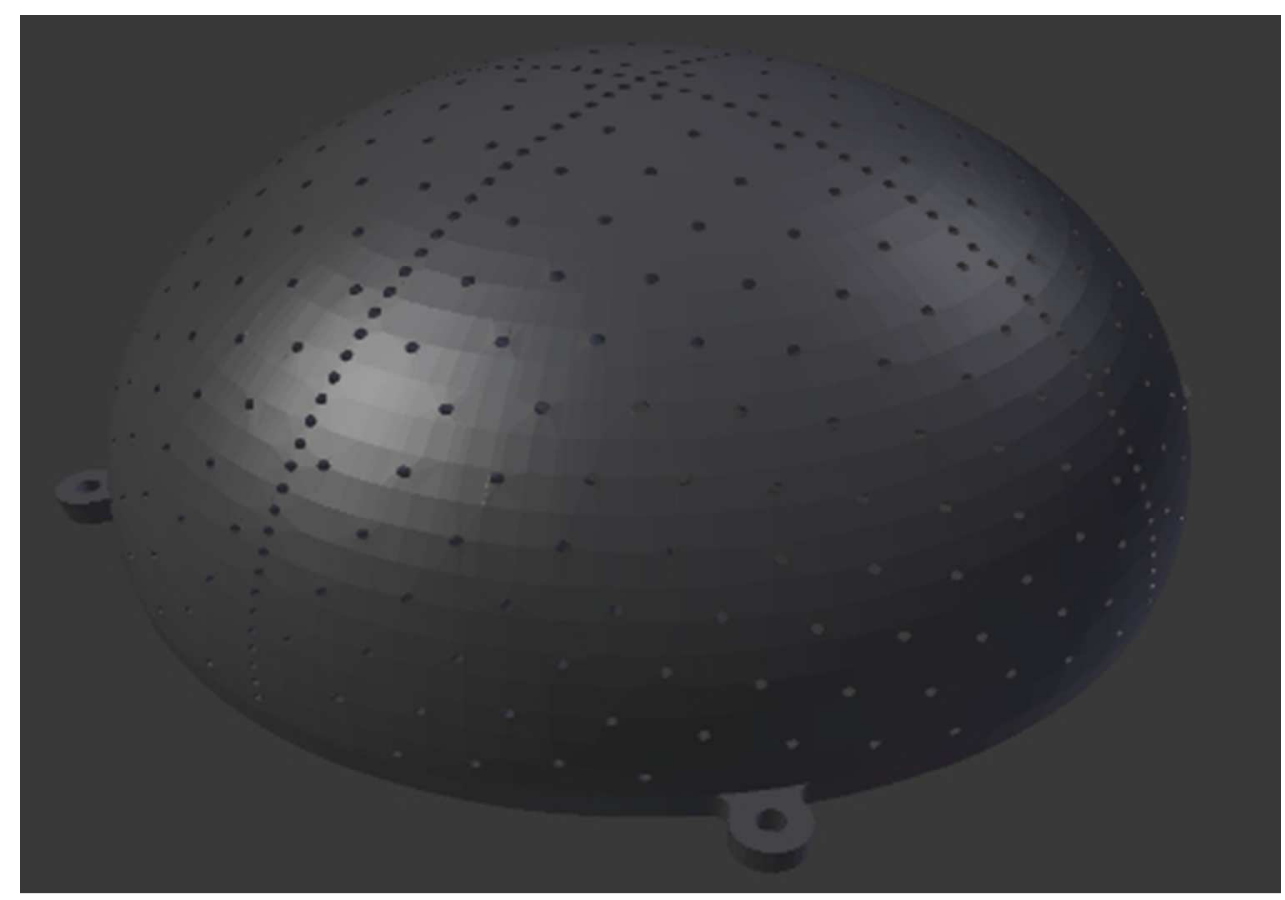




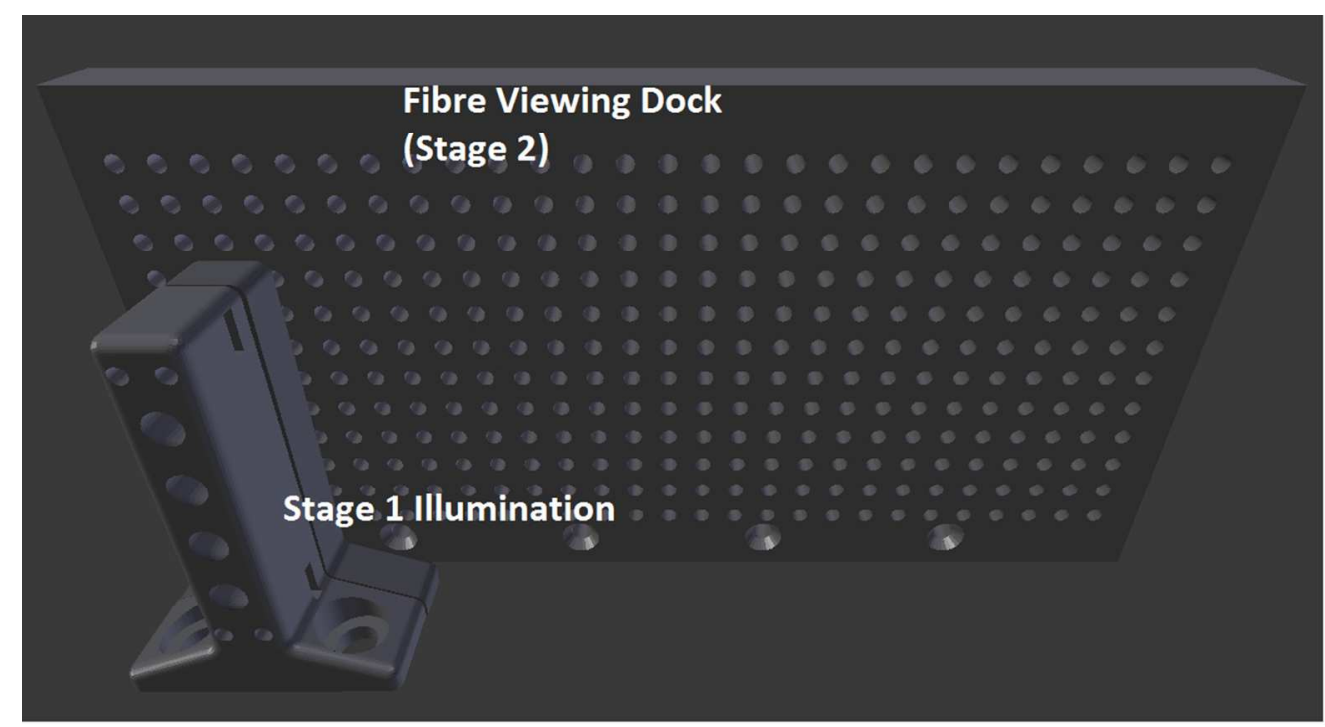




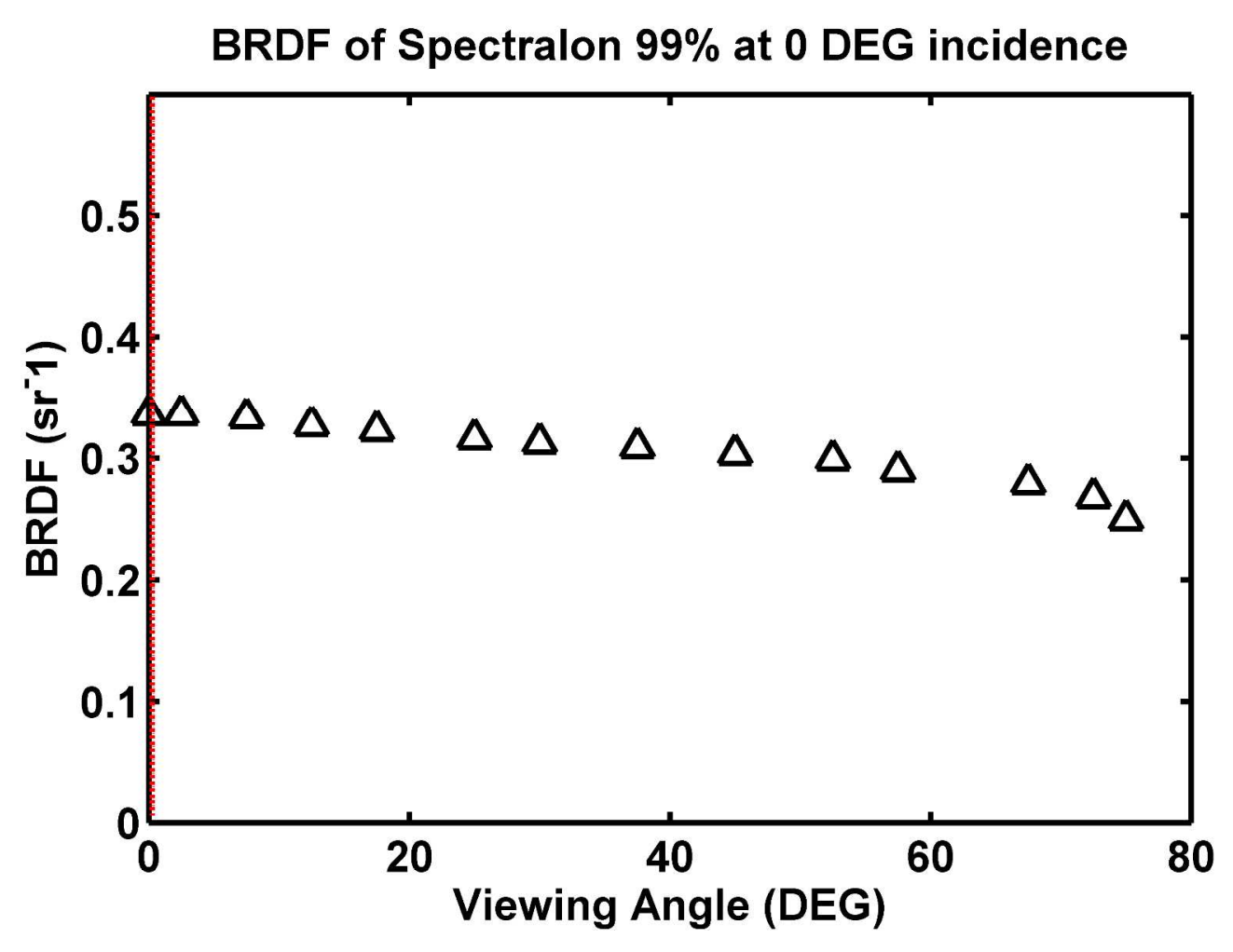




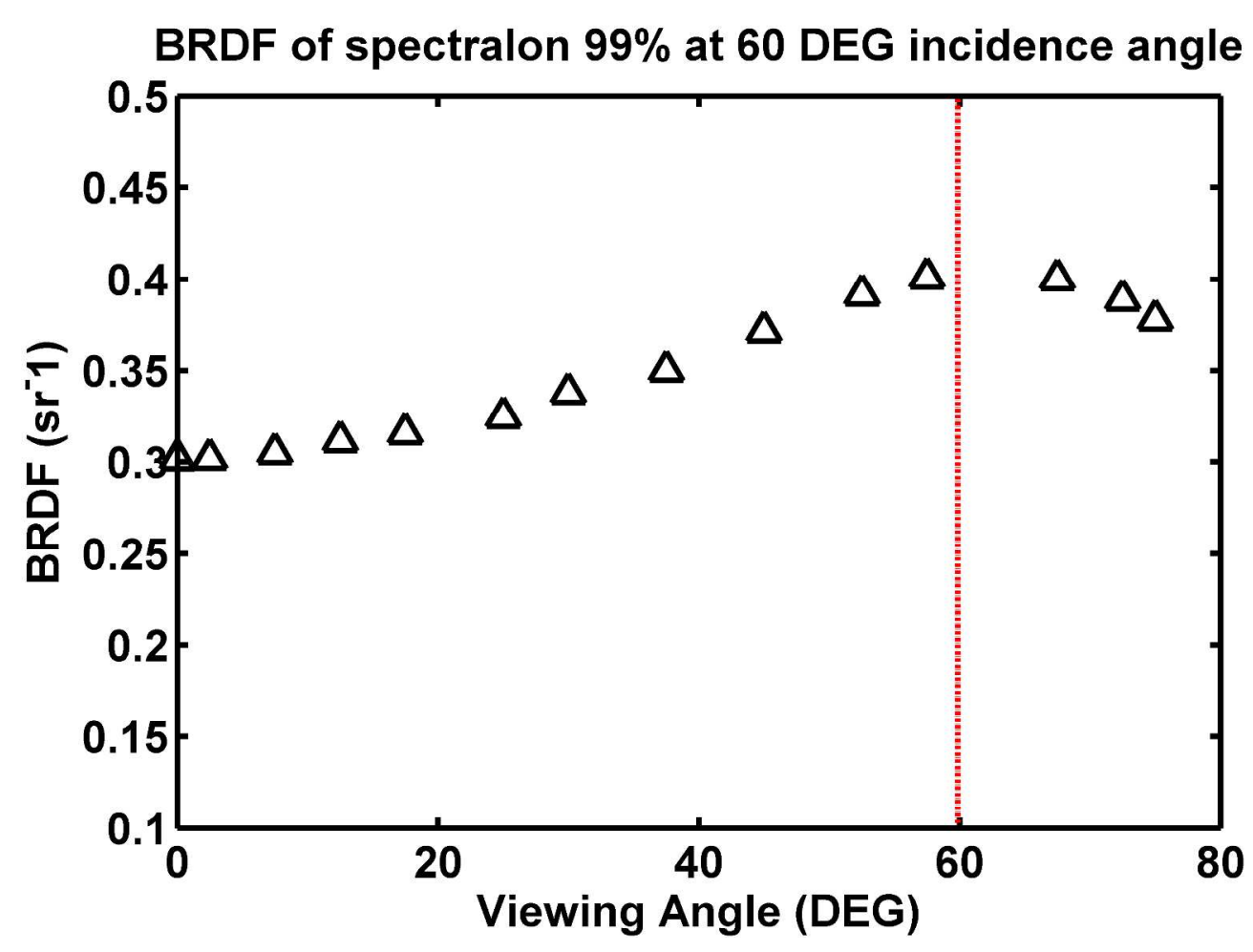




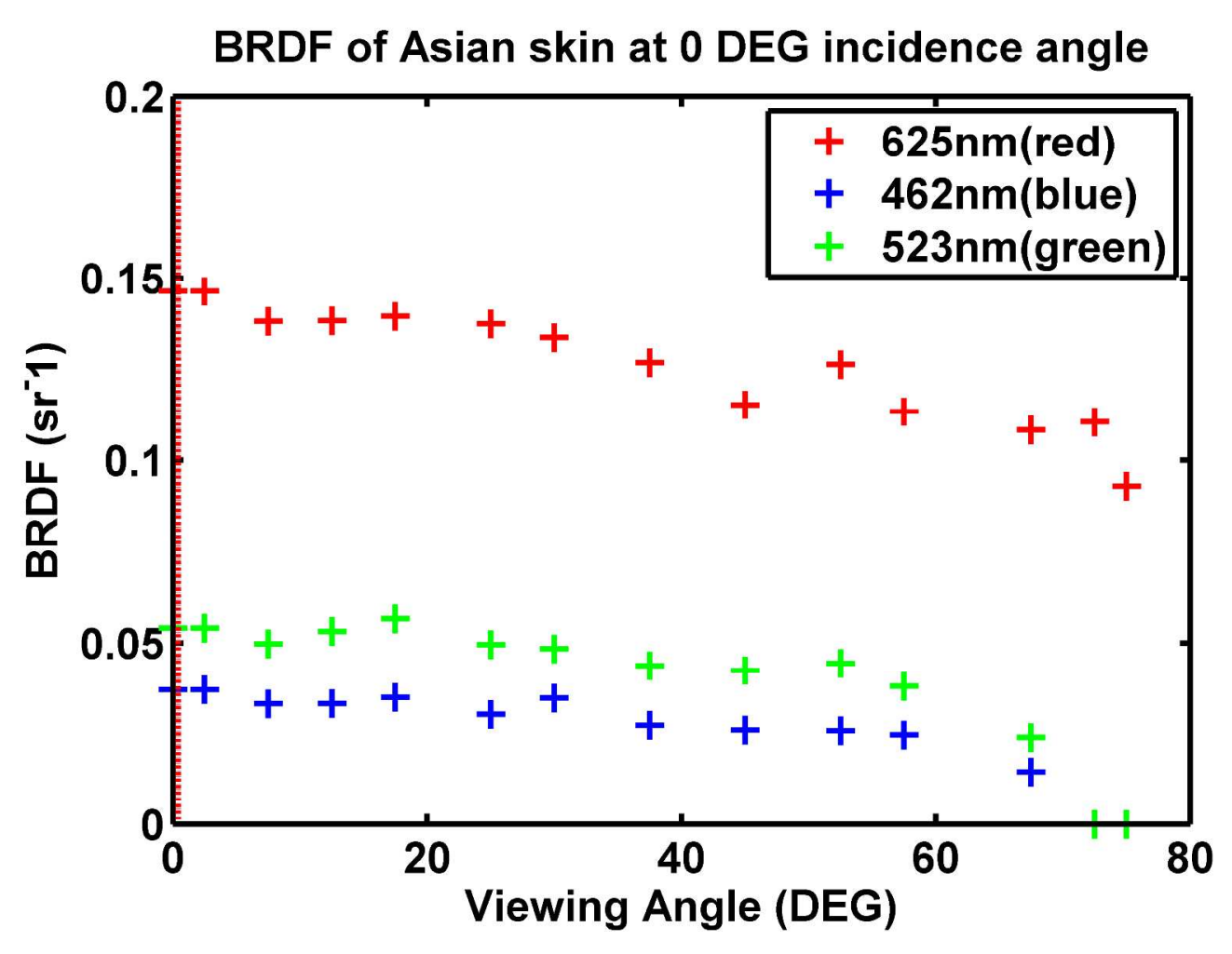




\section{BRDF of Asian skin at 60 DEG incidence angle}











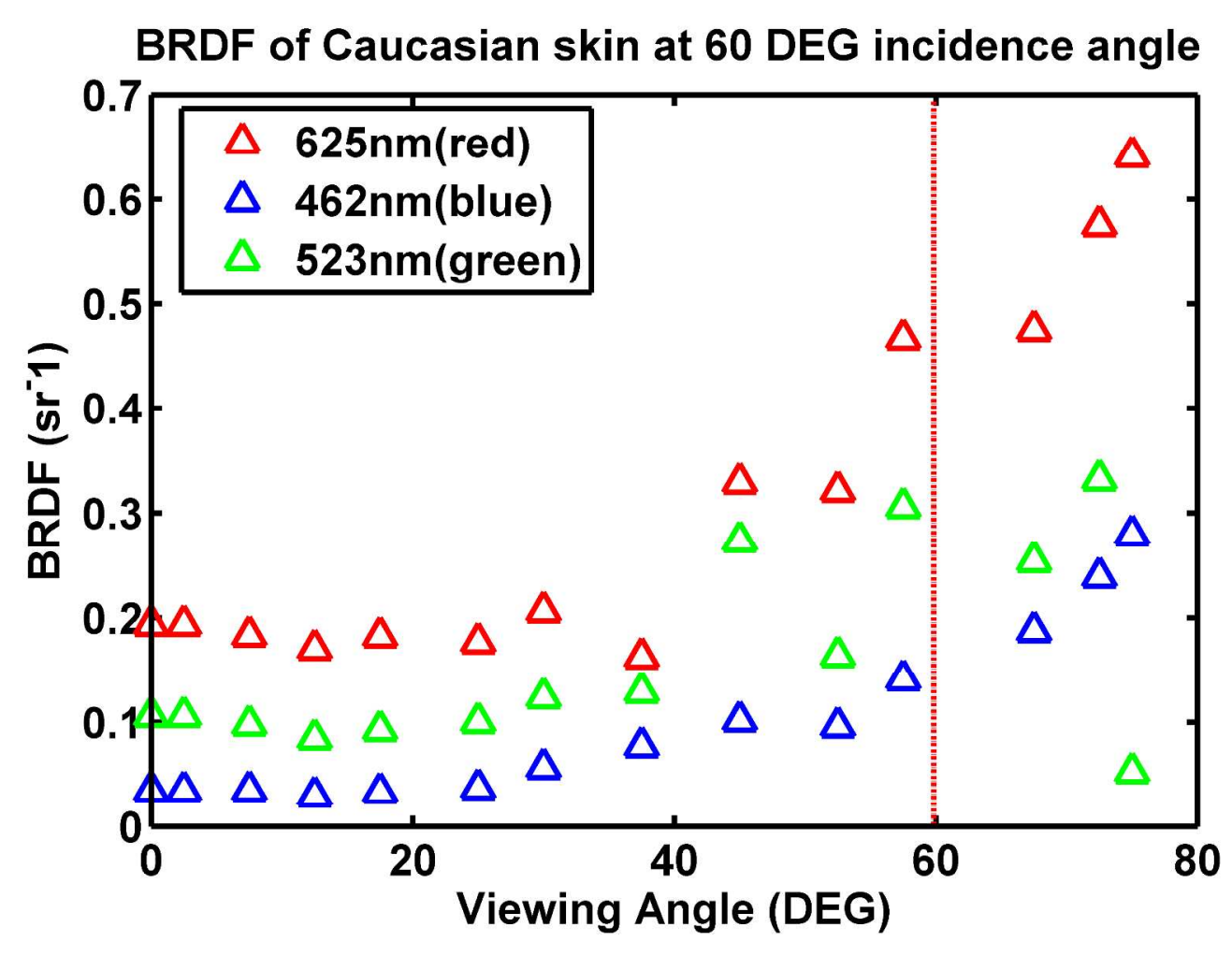

